\title{
Die vier Konferenzen des Europarates zur Revision der Lehrbücher in Geographie
}

Hans Bernhard

Die ersten beachtenswerten Ansätze zur Schulbuchrevision liegen zwischen den beiden Weltkriegen. Erwähnt sei der Vorstoß von Anatole France, der sich im Jahre 1919 auf einem Kongreß der französischen Volksschullehrer gegen alle Bücher wandte, die «den $\mathrm{Haß}$ predigen». Wenige Jahre später wurden in Frankreich 26 Schulbücher aus dem Handel gezogen oder im Sinne der obigen Postulation abgeändert. Mitten im Zweiten Weltkrieg fanden zwischen der USA und Kanada bilaterale Schulbuchbesprechungen statt, verbunden mit der kritischen Sicht einer großen Zahl von Vorlagen und mit entsprechenden Verbesserungsvorschlägen. Nach Beendigung des Krieges waren es vor allem internationale Organisationen wie Unesco und Europarat, die sich mit dieser Aufgabe befaßten. Natürlich sind nicht die Schulbücher aller Fächer in gleichem $\mathrm{Maße}$ anfechtbar. In allererster Linie sind es diejenigen aus dem Geschichts- und Geographieunterricht.

Deshalb veranstaltete der Europarat in der Zeit von 1953 bis 1958 vorerst sechs internationale Symposien zur Revision der Geschichtsbücher und etwas später vier weitere Konferenzen, die sich mit der Verbesserung der Geographiebücher befaßten und über die im folgenden etwas eingehender berichtet werden soll.

Die Tagungsorte waren so gewählt, daß sie jeweils in einem der zu besprechenden Großräume Mittel-, Süd-, West- und Nordeuropa lagen, 1961 in Goslar, 1962 in Santa Cruz/Teneriffa, 1963 in Bray/Irland und 1964 in Reykjavik. Die einzelnen Tagungen dauerten 10 bis 14 Tage. Darin waren in der Regel eine ganz- und eine halbtägige Exkursion, die die Teilnehmer mit der geographischen Eigenart der näheren Umgebung vertraut machen sollte, eingeschlossen. Nicht alle Mitgliedstaaten hatten das Recht, gleichviele Delegierte zu entsenden. Mit je drei Delegierten waren die Deutsche Bundesrepublik, Frankreich und Italien vertreten, mit zwei Delegierten Österreich, Belgien, die Niederlande und Griechenland und mit je einem Delegierten Dänemark, Norwegen, Schweden, Irland, Luxemburg und die Schweiz ${ }^{1}$.

Die Konferenzteilnehmer setzten sich aus Mittelschullehrern, Rektoren, Inspektoren und Hochschullehrern, zu einem schönen Teil Lehrbuchautoren, zusammen. Dazu kamen Beobachter von der Unesco, vom Europakolleg in Brügge, der Eu- ropaschule der Montanunion in Luxemburg und in Reykjavik gleich zwei Vertreter aus Finnland. Zypern als reguläres Mitglied war an keiner der Konferenzen vertreten. Dagegen hatte Portugal zu den beiden letzten Konferenzen einen Beobachter entsandt.

Das Ziel der Konferenzen bestand einmal in der Abfassung von Richtlinien und Fakten, die zukünftigen Lehrbuchautoren helfen sollten, ihr eigenes Land sowie andere europäische Räume aktuell, objektiv aus nationaler und übernationaler (europäischer) Sicht darzustellen. Der Europagedanke stand bei diesen Konferenzen nicht (wie vielleicht zu erwarten gewesen wäre) im Vordergrund, wenn auch einige Punkte der Fragebogen in dieser Richtung liefen.

Den Gang der Tagungen sollten vorbereitete Fragen erleichtern. Diese wurden den Delegierten schon vor Konferenzbeginn zugestellt und mußten in englischer oder französischer Sprache beantwortet und die Antworten vervielfältigt eingesandt werden. So lautet z. B. der Fragebogen für die dritte Konferenz in Bray:

1. Behandeln ihre Geographieschulbücher die Länder Europas nach großen Regionen? Wenn ja, welches sind die Namen dieser Regionen?

2. Wenn die Bezeichnung Nordwesteuropa verwendet wird, welches sind die Grenzen dieser Region und welche Länder gehören ganz oder teilweise dazu? Welches sind die gemeinsamen Merkmale dieser Region?

3. Wenn die Bezeichnung Nordwesteuropa nicht gebraucht wird, welche Bezeichnung wird für dieses Gebiet verwendet, welches Großbritannien, Irland, Frankreich, Belgien, die Niederlande und Luxemburg umschließt? Warum wurde diese Bezeichnung gewählt? Welches sind die Grenzen der so bezeich . neten Region?

4.a) Behandeln ihre Bücher genügend aus geographischer Sicht die ökonomischen, politischen und sozialen Beziehungen, welche diese nordwesteuropäischen Länder haben:

1. zu einander,

2. mit anderen Ländern Europas,

3. mit anderen Ländern außerhalb Europas?

b) Im besonderen wird befriedigend behandelt

1. die Beziehung der EWG zu den betreffenden Ländern, 
2. die Beziehung Großbritanniens und Nordirlands mit anderen Ländern des Commonwealth?

5. Erwähnen ihre Schulbücher

a) die Differenzen zwischen der geographischen Eigenart, welche Großbritannien und Nordirland gestaltet?

b) die politische Teilung in Irland?

6. a) Wie sind die Lehrbücher illustriert, um die Mannigfaltigkeit der Landschaft und die Verschiedenheit der ökonomischen Tätigkeit zu zeigen?

b) In welcher Art sind in den Lehrbüchern die Lokalbezeichnungen von Ortsnamen angewendet?

7. a) Beschränkt sich die Anthropogeographie ihrer Lehrbücher auf die Besprechung der Wirtschaftsgeographie im engeren Sinne oder ist sie bestrebt, die historisch-ökonomischen, die politischen und sozialen Faktoren zu prüfen und die kulturellen und sprachlichen Differenzen, welche innerhalb der nationalen Grenzen existieren, anzudeuten?

b) Ist diese Behandlung objektiv und ausgeglichen und trägt sie den Veränderungen der letzten zehn Jahre voll Rechnung?

8. Welchen Platz nimmt die Geographie in den Programmen der verschiedenen Schultypen ihres Landes ein? Ist dieses Fach auf allen oder nur auf einzelnen Stufen fakultativ oder obligatorisch? Welches ist in jedem Fall die ungefähre wöchentliche Stundenzahl?

9. Welches sind die größten Maßstäbe der verwendeten Karten von Nordwesteuropa, außer denjenigen der Atlanten?

10. In welchem Ausmaß werden visuelle und audiovisuelle Hilfsmittel wie Filme, Filmbänder, Dias, graphische Darstellungen, Modelle, Radio, Television, Grammophon und Tonband verwendet?

11. Existieren in Ihrem Lande Pläne in Hinsicht auf den Europarat, den Geographieunterricht auf europäischer Basis zu vertiefen und zu erweitern?

Aus diesem Fragenkomplex (und das gilt auch für die drei anderen) ist einmal ersichtlich, daß man sich mit der physiogeographischen Materie so gut wie nicht befaßte (außer der kritischen Durchsicht der Lehrbücher und natürlich des Wörterbuches) und daß bereits auch methodische Fragen und solche über die Bedeutung der Geographie im Fächersystem der Mittelschule miteinbezogen wurden.

Die Arbeit der Delegierten vollzog sich in Gesamtkonferenzen und in drei vorbereitenden Untergruppen, kurz als Komitee $1-3$ bezeichnet. Als Beispiel diene wieder Bray:

Komitee 1 beschäftigte sich mit der Definition und dem Inhalt des Begriffes Westeuropa (vorerst als Nordwesteuropa vorgeschlagen), der Darstellung seiner natürlichen Ausstattung und seiner wirtschaftlichen Entfaltung.
Komitee 2 befaßte sich mit der kartographischen Darstellung dieser Region. Jeder Teilnehmer hatte von allen Atlanten und Lehrbüchern seines Landes, die sich mit diesem Gebiet befaßten, je fünf Exemplare zur kritischen Durchsicht eingesandt. Unter anderem wurde die Zahl der Karten festgestellt, die sich mit Westeuropa und den zugehörigen Ländern befaßten, absolut und in Prozent aller Atlasblätter. Es bestanden natürlich recht große Unterschiede zwischen den einzelnen Atlanten, sowohl allgemein wie auch in der Aufgliederung in die verschiedenen Themakarten (geologische, klimatische, wirtschaftliche usw.) Auch Fehler, unkonsequente Anwendung von Signaturen und Schreibweisen und das Fehlen aktueller Neuerungen wurden festgestellt. Insgesamt standen in Bray 24 verschiedene Atlanten zur Verfügung, von der Schweiz diejenigen von Imhof (Schweizerischer Mittelschulatlas) und Kümmerly \& Frey (Schweizerischer Schulatlas). Ersterer wurde in der ersten Konferenz sogar als das «Nonplusultra» bezeichnet, später aber immer mehr vom österreichischen Atlas Hölzels «überschattet».

Komitee 3, für das ich mich eingeschrieben hatte, erarbeitete ein Wörterbuch mit geographischen Fachausdrücken in sechs Sprachen (deutsch, französisch, englisch, italienisch, niederländisch und spanisch). Kollege Quencez von der Europaschule in Luxemburg hatte es schon gut vorbereitet. Unzählige Begriffe aus dem Gesamtgebiet der Geographie waren zu bereinigen und übersichtlich in ein System einzuordnen. Dabei ergaben sich zwischen der Schweiz, Österreich und Deutschland besonders in physiogeographischer Hinsicht derart viele Unklarheiten, daß sie auf einer Sonderkonferenz in München bereinigt werden mußten.

Die Ergebnisse und Vorschläge wurden in der Gesamtversammlung besprochen, genehmigt oder abgeändert. Eine Simultananlage mit zwei Dolmetschern, die in den einzelnen Konferenzen mehr oder weniger gut funktionierte, ermöglichte die Verständigung und Zusammenarbeit.

Nicht nur Atlanten, sondern auch Lehrbücher, die den zur Sprache kommenden Teil Europas enthielten, mußten in je fünf Exemplaren eingesandt werden. Sie wurden von den Delegierten der betreffenden Länder durchgesehen und kritisch besprochen und zusammen mit den Atlanten während der Konferenzen ausgestellt.

Hier schnitt die Schweiz allerdings recht kläglich ab, denn mit unseren Geographiebüchern konnten wir uns mit den anderen Ländern in keiner Weise messen. In Bray waren Österreich und Belgien mit je 2, die Türkei mit 3, die Niederlande mit 5, Irland mit 6, Italien mit 7, England mit 8, die Bundesrepublik Deutschland mit 11, Frankreich mit 12 und Dänemark sogar mit 13 verschiedenen Geographiebüchern über Westeuropa vertreten, während für die Schweiz nur Süd- und Westeuropa von Anna- 
heim-Brunner und das vergriffene Buch "Europa» von Boss und Staub vorlagen. Aber gerade diese Beispiele zeigen, daß auch kleine Länder in der Schaffung von Lehrbüchern nicht zurückstehen müssen. Eigentlich hätte noch eine fünfte Konferenz mit dem Thema Osteuropa und Europa als Ganzes stattfinden sollen. Da der Europarat aber nicht über die notwendigen Geldmittel verfügte (Unterkunft und Verpflegung der Teilnehmer gingen auf Kosten desjenigen Staates, auf dessen Boden die Tagungen jeweils stattfanden), mußte auch das noch in Reykjavik erledigt werden.

Welches sind nun die Ergebnisse dieser vier Konferenzen? In dieser Art ist die Frage vielleicht etwas gewagt oder zumindest verfrüht. Wohl liegen Teilergebnisse vor. Dazu kommen verschiedene Vorschläge und Pläne teils realer, teils wenigstens für den Moment mehr programmatischer Art.

Ähnlich wie bei den Historikern sollen einige Publikationen erscheinen. Bereits liegen die Schlußrapporte aller Konferenzen vor. Sie befassen sich in erster Linie mit der Materie und dem Ablauf der einzelnen Tagungen. Wichtiger ist das Vocabularium Geographicum. Es umfaßt rund 300 Seiten und ist, wie bereits erwähnt, sechssprachig. Es enthält 3100 wesentliche Begriffe aus dem Gesamtgebiet der Geographie. Physio- und Anthropogeographie gliedern sich in 40 Begriffskategorien, wie Vulkanismus, Karstformen, Tiergeographie, Stadtgeographie usw., diese wieder in 617 Oberbegriffe. Am Schlusse findet sich ein alphabetisches Register in allen sechs Sprachen mit zugehöriger Seitenzahl. Allein schon dieses Werk, das in jeder Hinsicht als gelungen bezeichnet werden kann, wird für jeden geographisch Schaffenden von großem Wert sein, sei es, um einen bestimmten Begriff zu eruieren oder ihn systemgerecht einzuordnen oder in eine der fünf Fremdsprachen zu übersetzen.

Schon in Goslar wurde der Wunsch ausgesprochen, jeder Mitgliedstaat möchte sobald wie möglich eine gedrängte Landeskunde mit den notwendigsten Angaben und dem neuesten statistischen Material in einer der beiden Kongreßsprachen herausgeben. Von den Niederlanden, der Türkei, von Island und Irland lagen bereits solche Bücher vor. Für die Schweiz konnte das von Kümmerly \& Frey herausgegebene "La Suisse, ce qu'il faut savoir» abgegeben werden.

Demnächst soll in einem Londoner Verlag eine $\mathrm{Pu}-$ blikation "L'Europe en images» erscheinen. Sie enthält typische und zeitgemäße Bilder aus jedem Teilnehmerland, schwarzweiß und zum Teil farbig, etwa zehn Bilder von den kleineren und etwa fünfzehn bis zwanzig für die größeren Länder. Für die Schweiz wurden dreizehn Bilder mit zugehörigem Kommentar eingesandt. Wie viele davon verwendet werden können, hängt in erster Linie vom verfügbaren Kredit ab. Diese Broschüre soll vor allem
Lehrbuchautoren auf gute Illustrationsquellen hinweisen.

Vorgesehen ist ferner eine Publikation betitelt «Les différents pays d'Europe, vu par leurs Géographes». Vorerst plante man nur eine kritische Durchsicht fremder Lehrbücher, um darin Irrtümer und Fehler über das eigene Land festzustellen. Für die Schweiz wurden (vom Deutschen Lehrbuchinstitut in Braunschweig zur Verfügung gestellt) Lehrbücher in deutscher, französischer, englischer, italienischer und niederländischer Sprache durchgesehen. Erfreulich und auffallend war immer wieder die relativ große Seitenzahl, die der Darstellung unseres Landes eingeräumt wurde, unterhaltend, oft aber auch haarsträubend die darin enthaltenen Fehler. Nun soll aber diese Zusammenstellung durch eine kurze Monographie jedes Landes ergänzt werden, eine Maßnahme, über die sich künftige Lehrbuchautoren besonders freuen werden.

Als Ergänzung zu den Atlaskarten soll von Zeit zu Zeit eine Mappe mit statistischen Karten Europas erscheinen, die immer wieder die neusten Tatsachen vermitteln können.

Und schließlich wird noch eine Veröffentlichung erscheinen, die manchem Geographielehrer, der sich über die Verhältnisse an ausländischen Schulen orientieren möchte, gute Dienste leisten wird; "L'enseignement de la Géographie dans les écoles primaires et secondaires d'Europe». Eine Stundentafel zu dieser Studie ist bereits in der «Geographica Helvetica» 1965/1 erschienen. Glücklicherweise sind in anderen Ländern die Verhältnisse ungleich günstiger als in der Schweiz mit ihrem Irrgarten föderalistischer Schulpläne.

Das alles wäre sicher schon eine reiche Ausbeute der vier Konferenzen des Europarates zur Revision der Lehrbücher in Geographie, vor allem, wenn man die vielen Schwierigkeiten organisatorischer, sprachlicher und methodischer Art kennt.

Doch wie viel wertvoller wäre der lebendige Kontakt zwischen Geographielehrern hüben und drüben! Bilaterale und multilaterale Orientierung und Verständigung und Zusammenarbeit über die Grenzen! Möglichkeiten hiezu gäbe es genug

- durch den Austausch von Lehrbüchern und Atlanten,

— durch Beschaffung von Quellenmaterial zur Abfassung von Schulbüchern,

- Manuskripte könnten vor ihrer Drucklegung abschnittweise den betreffenden Partnern zur kritischen Durchsicht zugestellt werden, ein Vorgehen, das schon während der Konferenzen mit Erfolg ausprobiert wurde,

- durch die Publikation von Namenlisten mit Experten und den Adressen verschiedener Institute, die den Autoren behilflich sein könnten. 
Vor allem aber wäre der persönliche Kontakt in Form von kurzen Kursen mit Exkursionen oder gar durch den Austausch von Lehrern wünschenswert, ein Postulat, dem sprachliche Schwierigkeiten gewisse Grenzen setzen.

Wertvoll wäre die Schaffung nationaler Zentren:

a) Informationszentren für neuzeitliches Material hinsichtlich Text und Illustration

b) Dokumentationszentren mit Unterrichtsmaterial verschiedenster Art (Lehrbücher, Filme, Dias, Karten, statistischen Tabellen usw.).

Ganz unabhängig von dieser Forderung hat E. Bugmann, der derzeitige Präsident des Vereins schweizerischer Geographielehrer, an der letztjährigen Tagung in Baden, einen ähnlichen Vorschlag gemacht. Sicher wäre das Geographische Institut der ETH, als gesamtschweizerischer Vertreter, dazu der geeignete Ort.

Gewissermaßen als Koordinationsstelle ist aber ein europäisches Zentrum notwendig. Von ihm aus müßte die Initiative zur Verbesserung der heutigen Verhältnisse ausgehen. Es müßte multilaterale $\mathrm{Zu}$ sammenkünfte und den Austausch von Unterrichtsmaterial organisieren, ein Jahrbuch herausgeben und eine Bibliothek mit allem wichtigen Material über Unterricht und Didaktik besitzen.

Manche dieser Vorschläge und Postulate sind sicher realisierbar, andere vielleicht etwas gewagt. Manches mag sich überschneiden oder wiederholen. Aber ein Anfang sollte gewagt werden. Das Deutsche Schulbuchinstitut in Braunschweig (Prof.
Eckert und Dr. Schüdekopf), das für die Konferenzen des Europarates von großem Wert war, ist ein sprechender Beweis dafür, was mit zielbewußter Planung erreicht werden kann. Wenn auch die Schweiz bei der Herausgabe von Lehrbüchern für den Geographieunterricht eine eher bedenkliche Rolle spielt, sollte sie doch, Vollmitglied des Europarates, als Informationszentrum nicht beiseite stehen. Die zentrale Informationsstelle für Fragen des Schul- und Bildungswesens (Dir. Egger) in Genf ist auch für uns eine wertvolle Institution. Sie hat aber ihre besondere Aufgabe und genügt für rein geographische Belange nicht.

Wir müssen selbst etwas unternehmen und vor allem mehr zusammenarbeiten. Geeint sind wir stärker und leistungsfähiger. Die Zukunft wird zeigen, wie notwendig das ist.

\section{Anmerkung}

1 Da die Schweiz zur Zeit des ersten Kongresses noch nicht Mitglied des Europarates war, der Bundesrat aber einen Beobachter an diese Konferenz entsenden wollte, wurde ich als damaliger Präsident des Vereins schweizerischer Geographielehrer, auf Vorschlag von Herrn Prof. Gutersohn, nach Goslar und später auch an die übrigen Konferenzen delegiert. In Teneriffa war die Schweiz bereits Vollmitglied. 